\title{
Overweight/obesity: it is only food and physical activity?
}

\begin{abstract}
It is generally accepted that to maintain a healthy weight and to minimize the risk of developing many diseases such as cardiovascular disease, diabetes, hypertension etc everyone, women and men, elderly and children, should combine a balanced diet that includes a variety of foods and an active lifestyle. However data from the World Health Organization has classified overweight and obesity as the fifth leading risk factor for global mortality1 while data from the NHANES survey data shows that more than 2 in 3 adults ( 70.2 percent) were considered to be overweight or have obesity. 2 Despite the food over-consumption and the lack of exercise there are many other factors that directly or indirectly lead to weight gain.
\end{abstract}

Volume 8 Issue 4 - 2018

\section{Evangelia Strongilou}

Clinical Dietitian, Nutritionist, Greece

Correspondence: Evangelia Strongilou, Clinical Dietitian, Nutritionist, Greece, Email estrongilou@hotmail.gr

Received: June 17, 2018| Published: July 06, 2018

\section{Opinion}

The main characteristic of overweight/ obesity is the higher energy intake than the energy expenditure. Some factors that are related with gain weight affect the energy consumption as they have been associated with higher calorie intake. These factors are:

a. The speed of eating: In a study in New Zealand the results showed that higher speed of eating is related with higher $\mathrm{BMI}^{3}$ while in a study in Japan the decreases in eating speeds can lead to reduction in BMI. ${ }^{4}$ This is something that may be related with the fact that when someone is eating fast may lead to the consumption of more food that is needed as he/she does not realize the time he/ she satiates.

b. Stress: Living in a demanding environment, trying to seize the time as efficiently as possible may lead not only in bad food choices, less time to work out but maybe can change normal functions of the body through production of hormones such as cortisone that contributes to gain weight. ${ }^{5,6}$

c. Inadequate sleep: The sleep duration is associated with the BMI. People who sleep less than 7-9 hours per night have higher BMI and have abdominal obesity according to a study that used data from the National Health and Nutrition Examination Survey (NHANES) 2005-2010. These findings are confirmed by many other studies that suggest sleep deprivation may be an independent risk factor of overweight/obesity. ${ }^{7-9}$

d. Chemical exposure: Many studies suggest that exposure, even from the pregnancy, in chemicals (including these that are correlated with smoking) may lead to obesity as these chemicals may alter the gene expression and promote the increased fat deposition in the body. ${ }^{10}$

e. Genes: Something that is related to overweight/ obesity is our genetic background. Some genes such as FTO are related with higher risk of developing an increased body weight but something that we should consider is that we can change our lifestyle in order to overcome this obstacle. ${ }^{11}$

f. Medical conditions and drugs: In this section we should include two aspects: medical conditions that may lead to weight gain especially if they are untreated such as polycystic ovary syndrome, hypothyroidism and some drugs that can affect the metabolism and cause an increased body weight. Such drugs are steroids, certain antidepressants, and medications to control seizures and psychiatric problems.12

\section{Conclusion}

Obesity is a multifactorial situation and many factors contribute to develop an excessive body weight. In general we should try to intervene and change the factors that we are able to affect. Having a balanced diet, a moderate physical activity, a life with less stress and adequate sleep and a healthy lifestyle in general is the key to maintain a healthy weight.

\section{Acknowledgments}

None.

\section{Conflict of interest}

The author declares there is no conflict of interest.

\section{References}

1. World Health Organization (WHO) Fact sheet $\mathrm{N}^{\circ} 311$.

2. Flegal KM, Kruszon-Moran D, Carroll MD, et al. Trends in obesity among adults in the United States, 2005 to 2014. JAMA. 2016;315(21):22842291.

3. Sook Ling Leong, Clara Madden, Andrew Gray, et al. Faster Self-Reported Speed of Eating Is Related to Higher Body Mass Index in a Nationwide Survey of Middle-Aged Women. J Am Diet Assoc. 2011;111(8):11921197.

4. Yumi Hurst, Haruhisa Fukuda. Effects of changes in eating speed on obesity in patients with diabetes: a secondary analysis of longitudinal health check-up data. BMJ Open. 2018;8:e19589.

5. Koski M, Naukkarinen H. The Relationship between Stress and Severe Obesity: A Case-Control Study. Biomed Hub 2017;2:458771.

6. Karen A Scott, Susan J Melhorn, Randall R Sakai. Effects of Chronic Social Stress on Obesity. Curr Obes Rep. 2012;1(1):16-25.

7. Knutson KL. Does inadequate sleep play a role in vulnerability to obesity? Am J Hum Biol. 2012;24(3):361-371. 
8. Ford ES, Li C, Wheaton AG, et al. Sleep duration and body mass index and waist circumference among US adults. Obesity 2014;22:598-607.

9. Girardin Jean-Louis, Natasha J Williams, Daniel Sarpong, et al Associations between inadequate sleep and obesity in the US adult population: analysis of the national health interview survey (1977-2009). BMC Public Health. 2014;14:290.
10. Wendee Holtcamp. Obesogens: An Environmental Link to Obesity. Environmental Health Perspectives. 2012;120(2):62-68.

11. Hinney A, Vogel CI, Hebebrand J. From monogenic to polygenic obesity: recent advances. Eur Child Adolesc Psychiatry. 2010;19:297-310.

12. Overweight and obesity: Causes and consequences. Centers for Disease Control and Prevention. 2012. 